\title{
NY-ESO-1 expression in synovial sarcoma and other mesenchymal tumors: significance for NY-ESO-1-based targeted therapy and differential diagnosis
}

\author{
Jin-Ping Lai ${ }^{1}$, Paul F Robbins ${ }^{2}$, Mark Raffeld ${ }^{1}$, Phyu Phyu Aung ${ }^{1}$, Maria Tsokos ${ }^{1}$, \\ Steven A Rosenberg ${ }^{2}$, Markku M Miettinen ${ }^{1}$ and Chyi-Chia Richard Lee ${ }^{1}$ \\ ${ }^{1}$ Laboratory of Pathology, National Cancer Institute, NIH, Bethesda, MD, USA and ${ }^{2}$ Surgery Branch, National \\ Cancer Institute, NIH, Bethesda, MD, USA
}

\begin{abstract}
A promising targeted therapy against NY-ESO-1 (CTAG 1B) using genetically modified T-cells in synovial sarcomas was recently demonstrated in a clinical trial at the $\mathrm{NCl}$. To investigate the role of NY-ESO-1 immunohistochemistry in patient selection and gain better insight into the incidence of NY-ESO-1 expression in synovial sarcomas and other mesenchymal tumors, we evaluated NY-ESO-1 expression by immunohistochemistry in 417 tumors. This collection of samples included: 50 SS18/SSX1/2 fusion positive synovial sarcomas, 155 gastrointestinal stromal tumors (GIST), 135 other spindle cell sarcomas as well as 77 other sarcomas (chondrosarcoma, osteosarcoma, dedifferentiated liposarcoma, alveolar soft part sarcoma, rhabdomyosarcoma, angiosarcoma, malignant mesothelioma, and Ewing's sarcoma). We report that $76 \%$ of synovial sarcomas expressed NY-ESO-1 in a strong and diffuse pattern $(2-3+,>50-70 \%$ of tumor cells). In contrast, only rare cases of other spindle cell mesenchymal tumor expressed NY-ESO-1 (GIST (2/155), malignant peripheral nerve sheath tumors (1/34), and dermatofibrosarcoma protuberans $(2 / 20))$. Individual cases of other sarcomas (angiosarcoma, malignant mesothelioma, chondrosarcoma, osteosarcoma, dedifferentiated liposarcoma, alveolar soft part sarcoma, and Ewing's sarcoma) were positive for NY-ESO-1. However, no positive cases were identified amongst our cohort of leiomyosarcomas (0/24), hemangiopericytoma/solitary fibrous tumors (0/40), and cellular schwannomas (0/17). In summary, we find that NY-ESO-1 is strongly and diffusely expressed in a majority of synovial sarcomas, but only rarely in other mesenchymal lesions. Beyond its role in patient selection for targeted therapy, immunohistochemistry for NY-ESO-1 may be diagnostically useful for the distinction of synovial sarcoma from other spindle cell neoplasms.

Modern Pathology (2012) 25, 854-858; doi:10.1038/modpathol.2012.31; published online 2 March 2012
\end{abstract}

Keywords: NY-ESO-1; sarcoma; targeted therapy

Cancer testis antigens have emerged over the last decade as both a diagnostic marker and a therapeutic target in malignant lesions. ${ }^{1}$ One example is the cancer testis antigen encoded by the CTAG $1 B$ gene, NY-ESO-1 (New York esophageal squamous cell carcinoma 1). Its aberrant expression has been observed in a variety of neoplasms, including esophageal carcinoma, hepatocellular carcinoma,

Correspondence: Dr C-CR Lee, MD, PhD, Laboratory of Pathology, National Cancer Institute/NIH, Building 10/2B50, 10 Center Dr, Bethesda, MD 20892, USA.

E-mail: leechy@mail.nih.gov

Received 23 September 2011; revised 6 December 2011; accepted 9 December 2011; published online 2 March 2012 melanoma, and synovial sarcoma. ${ }^{1-4}$ In an analysis of synovial sarcomas, a large proportion, 80\% (20/ 25), demonstrated NY-ESO-1 immunoreactivity. ${ }^{4} \mathrm{~A}$ recent clinical trial conducted at the National Cancer Institute demonstrated that a T-cell receptor (TCR)-based gene therapy directly against NY-ESO-1 was a promising therapeutic modality for patients with synovial sarcoma with objective clinical responses observed in four of six patients refractory to all standard therapy. ${ }^{1}$ These recent advances support the need for the screening of additional cohorts of synovial sarcomas for NY-ESO-1. In addition, it raises the possibility that patients with other NY-ESO-1-expressing mesenchymal neoplasms may benefit from NY-ESO-1 targeted therapy, and finally 
that the differential expression of NY-ESO-1 may be useful to distinguish synovial sarcoma from other mesenchymal tumors.

To address these possibilities, we examined NYESO-1 expression by immunohistochemistry in cohorts of mesenchymal neoplasms including synovial sarcomas, gastrointestinal stromal tumor, leiomyosarcoma, malignant peripheral nerve sheath tumor, cellular schwannoma, dermatofibrosarcoma protuberans, hemangiopericytoma/solitary fibrous tumor, angiosarcoma, malignant mesothelioma, chondrosarcoma, osteosarcoma, dedifferentiated liposarcoma, and Ewing's sarcoma. We find that NY-ESO-1 is most consistently expressed in synovial sarcomas with rare exceptions in other sarcomas examined.

\section{Materials and methods}

\section{Tumors}

Our cohort of tumor samples include 50 stage IV synovial sarcomas that were positive for the SS18/ SYT gene rearrangement (SS18/SSX1/2 fusion), 155 gastrointestinal stromal tumors (GIST), 34 malignant peripheral nerve sheath tumors, 20 leiomyosarcomas, 40 hemangiopercytoma/solitary fibrous tumors, 20 dermatofibrosarcoma protuberans, 17 cellular schwannomas, 20 angiosarcomas, 18 Ewing's sarcomas, 27 malignant mesothelioma, and 12 other sarcomas including osteosarcoma, ${ }^{4}$ chondrosarcoma, ${ }^{2}$ dedifferentiated liposarcoma, ${ }^{1}$ alveolar soft part sarcoma, ${ }^{1}$ undifferentiated sarcoma, ${ }^{1}$ high-grade spindle cell sarcoma, ${ }^{2}$ and rhabdomyosarcoma. ${ }^{1}$

\section{Immunohistochemistry}

Paraffin-embedded tissue sections $(5 \mu \mathrm{m})$ were deparaffinized through xylene and graded alcohols. Immunohistochemical staining for NY-ESO-1 (Invitrogen, 1:100) was performed following heat-induced epitope retrieval, using target retrieval solution, low $\mathrm{pH}$ (DAKO). ${ }^{5}$ Slides were incubated in Tris with $3 \%$ goat serum for $15 \mathrm{~min}$ and then incubated for $1-2 \mathrm{~h}$ at room temperature with primary antibody. Detection was carried out using an automated slide stainer (DAKO; Autostainer) with a horseradish peroxidase/3,3'-diaminobenzidine polymer-based detection system (DAKO; Envision +$)$. Standard tissue sections of 50 cases of synovial sarcomas, 54 cases of gastrointestinal stromal tumors, and 12 other mesenchymal neoplasms were stained for NY-ESO-1. The remaining cases of GIST $(n=101)$ and other types of mesenchymal neoplasms $(n=200)$ were performed on tissue arrays. Arrays were constructed by using $6-10 \mathrm{~mm}^{2}$ of representative lesional tissue from each case with 20-60 specimens per slide. ${ }^{6}$ NY-ESO-1 staining intensity was assessed by at least two pathologists (CL, MM, JL, and MT), using the following scoring system: $1+=$ weak, $2+=$ moderate, and $3+=$ strong. The percentage of stained lesional cells was estimated for each case. A consensus opinion was achieved for any discordance between the pathologists $(<5 \%$ of cases).

\section{Statistics}

The GraphPad Software for Fisher's exact $P$ value was used to calculate the two-tailed $P$ value.

\section{Results}

We assessed the histological features of the 50 cases of translocation-confirmed stage IV synovial sarcomas. Forty-six tumors had a monophasic, spindle cell morphology, whereas the remaining four cases were biphasic tumors with scant epithelioid component admixed with spindle cells. Immunohistochemical staining for NY-ESO-1 expression revealed a diffuse staining pattern $(2-3+,>50 \%$ tumor cells) in $76 \%$ of the cases (38/50) (Figures 1a and b), three cases showed weak and focal expression $(1+,<10 \%$ of lesional cells), and nine cases were negative for NY-ESO-1 (Table 1). In all four biphasic lesions, both the spindle and epithelioid components were positive for NY-ESO-1.

In contrast, only three cases of the other mesenchymal lesions (3/135) stained were positive for NYESO-1. These included one case of malignant peripheral nerve sheath tumor (1/34) and two cases of dermatofibrosarcoma protuberans (2/20). However, we did not identify any cases of leiomyosarcoma (0/ 24), cellular schwannoma (0/17), or hemangiopericytoma/solitary fibrous tumors (0/40) that expressed NY-ESO-1. (Figures 1c and d; and Table 1). Thus, the incidence of NY-ESO-1 expression in synovial sarcomas was significantly higher than that in other spindle cell neoplasms examined in this study $(P<0.0001)$.

Of the 155 cases of GIST including gastric GISTs $(n=87)$, intestinal GISTs $(n=53)$, and metastatic GISTs involving the liver or mesentery $(n=15), 98 \%$ $(151 / 155)$ of the tumors evaluated were negative for NY-ESO-1 expression. Amongst the cases that showed some degree of expression was one case of gastric GIST $(3+,>90 \%$ tumor cells), one case of intestinal GIST $(2-3+, 50 \%$ tumor cells), and two cases of gastric GISTs that showed minimal NY-ESO1 staining (weak staining in $<2 \%$ of tumor cells). The incidence of NY-ESO-1 expression in gastrointestinal stromal tumor is significantly lower than that in synovial sarcoma $(P<0.0001)$ (Figure 2 and Table 1). These findings support the utility of NYESO-1 expression in the distinction between synovial sarcomas and GISTs $(P<0.0001)$.

Among the 77 other mesenchymal tumors analyzed, 93\% of tumors (72/77) were negative for NYESO-1 by immunohistochemistry, including Ewing's sarcoma (0/18), malignant mesothelioma (0/27), and 

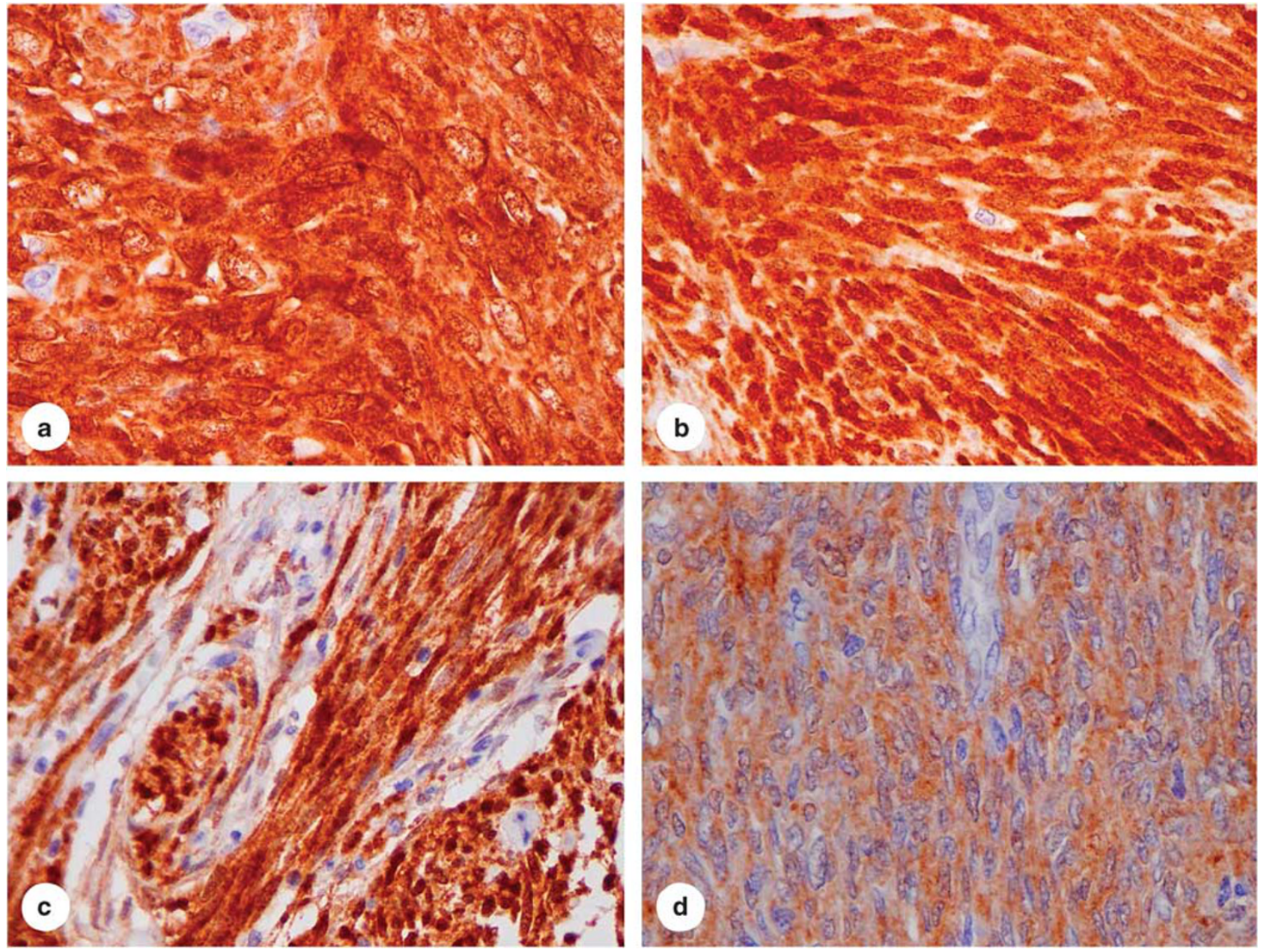

Figure 1 NY-ESO-1 expression patterns in synovial sarcomas, malignant peripheral nerve sheath tumors, and dermatofibrosarcoma protuberans $(40 \times)$ : (a and b), strong and diffuse expression $(2-3+,>50 \%$ of tumor cells) in synovial sarcomas (38/50) of biphasic (4)(a) and monophasic (46)(b) subtypes; (c), strong and diffuse expression $(2-3+,>50 \%$ of tumor cells) in malignant peripheral nerve sheath tumors (1/34); and (d), moderate and diffuse expression $(2+,>50 \%$ of tumor cells) in dermatofibrosarcoma protuberans $(2 / 20)$.

osteosarcoma (0/4). Expression of NY-ESO-1 was seen in $10 \%$ of angiosarcomas $(2 / 20)$, one chondrosarcoma (1/2), one dedifferentiated liposarcoma (1/1), and one undifferentiated sarcoma (1/1). However, considering the small number of samples tested in the latter entities (chondrosarcoma, dedifferentiated liposarcoma, and undifferentiated sarcoma), additional cases need to be evaluated to better determine the frequency of NY-ESO-1 expression in these sarcomas.

\section{Discussion}

Recently, a TCR-based gene therapy targeting NYESO-1 was demonstrated to be effective in mediating tumor regression in patients with metastatic synovial sarcoma. ${ }^{1}$ Thus, it has emerged that pretreatment evaluation of NY-ESO-1 expression in synovial sarcomas by immunohistochemistry is critical for treatment decisions. Additionally, the strong expression of NY-ESO-1 in the vast majority of synovial sarcomas suggests a potential use of this
Table 1 Positive NY-ESO expression distinguishes synovial sarcoma from GISTs and other types of sarcomas

\begin{tabular}{|c|c|c|c|c|}
\hline & $\mathrm{N}$ & $\begin{array}{l}\text { NY-ESO } \\
\text { positive }\end{array}$ & $\begin{array}{l}\text { NY-ESO } \\
\text { negative }\end{array}$ & \\
\hline Synovial sarcomma & 50 & $41(82 \%)$ & 9 & \\
\hline GISTs & 155 & $2(1 \%)$ & 153 & $P<0.0001$ \\
\hline Leiomyosarcoma & 24 & $0(0 \%)$ & 24 & $P<0.0001$ \\
\hline MPNST & 34 & $1(3 \%)$ & 33 & $P<0.0001$ \\
\hline SFT & 40 & $0(0 \%)$ & 40 & $P<0.0001$ \\
\hline $\begin{array}{l}\text { Cellular } \\
\text { schwannoma }\end{array}$ & 17 & $0(0 \%)$ & 17 & $P<0.0001$ \\
\hline DFSP & 20 & $2(10 \%)$ & 18 & $P<0.0001$ \\
\hline Angiosarcoma & 20 & $2(10 \%)$ & 18 & $P<0.0001$ \\
\hline Ewing sarcoma & 18 & $0(0 \%)$ & 18 & $P<0.0001$ \\
\hline $\begin{array}{l}\text { Malignant } \\
\text { mesothelioma }\end{array}$ & 27 & $0(0 \%)$ & 27 & $P<0.0001$ \\
\hline Other sarcomas ${ }^{\mathrm{a}}$ & 12 & 3 & 9 & $P=0.0003$ \\
\hline
\end{tabular}

Abbreviations: GIST: gastrointestinal stromal tumor; MPNST: malignant peripheral nerve sheath tumor; SFT: hemangiopericytoma/ solitary fibrous tumor; DFSP: dermatofibrosarcoma protuberans.

${ }^{a}$ Others sarcoma includes osteosarcoma (0/4), chondrosarcoma (1/2), dedifferentiated liposarcoma (1/1), alveolar soft part sarcoma (0/1), undifferentiated sarcoma (1/1), high-grade spindle cell sarcoma $(0 / 2)$ and rhabdomyosarcoma $(0 / 1)$. 

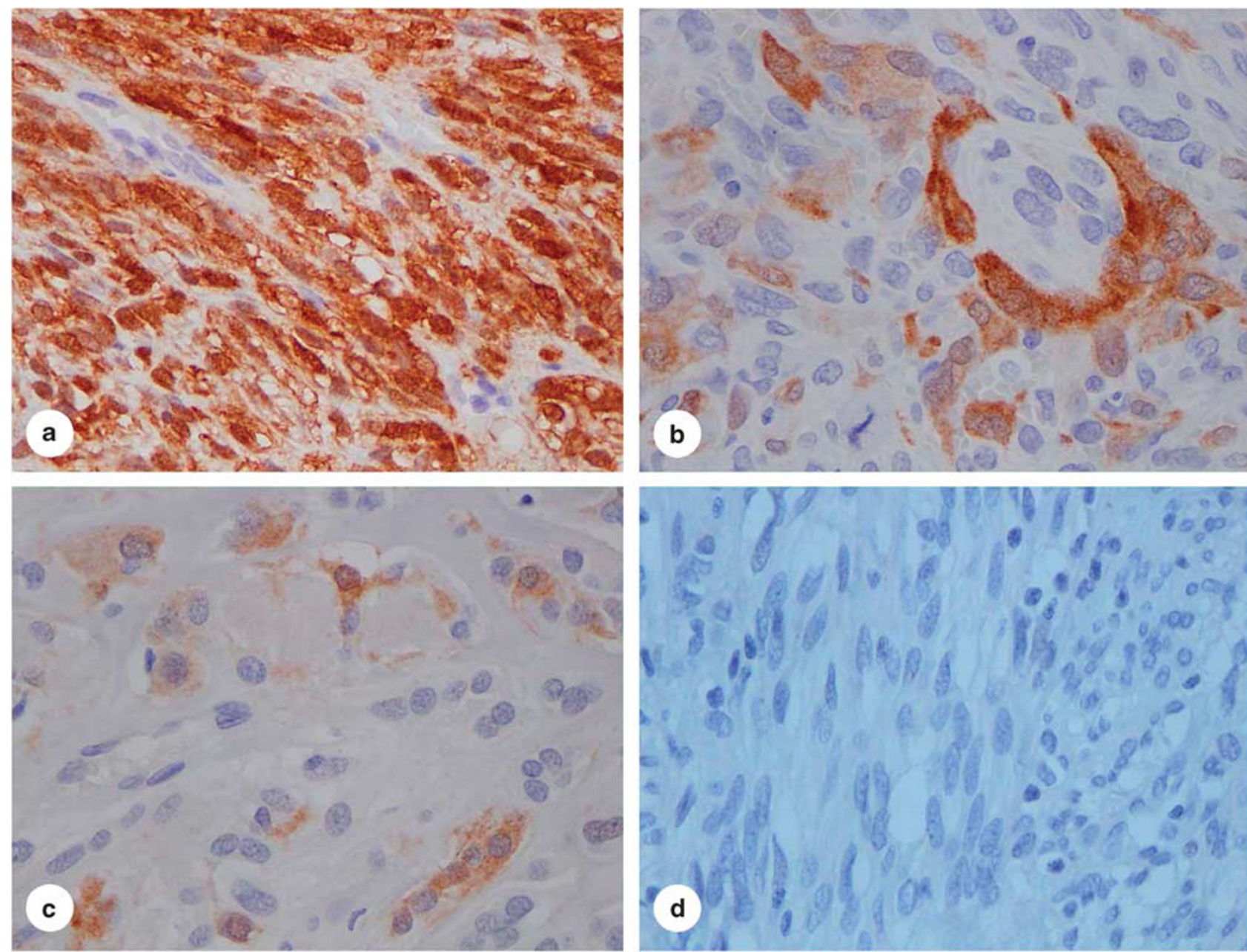

Figure 2 NY-ESO-1 expression in gastrointestinal stromal tumor $(40 \times)$ : (a), strong and diffuse expression $(3+,>90 \%$ of tumor cells) in gastrointestinal stromal tumors (GIST) (1/155); (b), focal and moderate expression ( $2+,<50 \%$ of tumor cells) in GIST (1/155); (c), focal and weak expression ( $1-2+,<2 \%$ of tumor cells) in two cases of GIST (2/155); (d), negative ( $0+, 0 \%)$ in 151 cases of GIST (151/155).

marker in the differential diagnosis of this malignancy. Though NY-ESO-1 expression was previously reported in $80 \%$ of synovial sarcomas, its utility in the diagnosis of synovial sarcoma was not pursued in the absence of large enough cohorts of other morphologically similar lesions. ${ }^{4}$ Our findings are consistent with this earlier report, and we found that $82 \%$ of synovial sarcomas are positive for NYESO-1 with $76 \%$ of synovial sarcomas expressing diffusely high levels of NY-ESO-1 $(2-3+,>50 \%$ tumor cells). We further utilize these findings to determine whether we could distinguish synovial sarcomas from other sarcomas. Our findings reveal that NY-ESO-1 was rarely expressed in sarcomas other than synovial sarcomas, with rare cases of malignant peripheral nerve sheath tumors and dermatofibrosarcoma protuberans positive for NYESO-1. Therefore, we conclude that NY-ESO-1 expression can reinforce a diagnosis of synovial sarcoma. Intriguingly, the rare cases of NY-ESO-1positive sarcomas other then synovial sarcomas suggest potential candidate lesions for NY-ESO-1targeted treatment, and will require further analysis of NY-ESO-1 expression in larger cohorts of these lesions.

To date, there have been only two studies examining expression of NY-ESO-1 in gastrointestinal stromal tumors. Perez et $a l^{7}$ reported NY-ESO-1 expression in 20\% (7/35 cases) of GIST. More recently, the same group found that expression of NY-ESO-1 was associated with tumor progression despite Imatinib treatment. ${ }^{8}$ In our study, only 2 of 155 GIST (1\%) showed high-level expression of NYESO- $1(2+, 50 \%$ tumor cells, and $3+,>90 \%$ of tumor cells, respectively), but these patients could be potential candidates for targeted therapy against NY-ESO-1 tumor antigen.

It may be difficult to distinguish monophasic spindled synovial sarcomas from other spindle cell tumors by histological examination alone (particularly in small biopsy specimens), and there are only a limited number of immunohistochemical markers that are helpful in differential diagnosis of these tumors. Results presented in this study demonstrate that, unlike synovial sarcomas, the vast majority of other sarcomas and spindle cell mesenchymal 
tumors do not express NY-ESO-1. Therefore, NYESO-1 is a potentially useful diagnostic marker for differentiating synovial sarcoma from other spindle cell type sarcomas and tumors, particularly cellular schwannoma, leiomyosarcoma, and dermatofibrosarcoma protuberans.

In conclusion, immunohistochemistry for NYESO-1 not only has a critical role in the selection of patients with synovial sarcomas for targeted therapy, but may also be used to distinguish synovial sarcomas from other spindle cell neoplasms, such as leiomyosarcoma, cellular schwannoma, and dermatofibrosarcoma protuberans. In addition, our findings of rare cases of other mesenchymal lesions that express NY-ESO-1 suggest the importance of future studies of these lesions.

\section{Disclosure/conflict of interest}

The authors declare no conflict of interest.

\section{References}

1 Robbins PF, Morgan RA, Feldman SA, et al. Tumor regression in patients with metastatic synovial cell sarcoma and melanoma using genetically engineered lymphocytes reactive with NY-ESO-1. J Clin Oncol 2011;29:917-924.

2 Mashino K, Sadanaga N, Tanaka F, et al. Expression of multiple cancer-testis antigen genes in gastrointestinal and breast carcinomas. Br J Cancer 2001;85:713-720.

3 Wang XY, Chen HS, Luo S, et al. Comparisons for detecting NY-ESO-1 mRNA expression levels in hepatocellular carcinoma tissues. Oncol Rep 2009;21:713-719.

4 Jungbluth AA, Antonescu CR, Busam KJ, et al. Monophasic and biphasic synovial sarcomas abundantly express cancer/testis antigen NY-ESO-1 but not MAGE-A1 or CT7. Int J Cancer 2001;94:252-256.

5 Tabe Y, Sebasigari D, Jin L, et al. MDM2 antagonist nutlin-3 displays antiproliferative and proapoptotic activity in mantle cell lymphoma. Clin Cancer Res 2009;15:933-942.

6 Miettinen M. A simple method for generating multiple blocks without special equipment. Appl Immunohistochem Mol Morphol 2012 (in press).

7 Perez D, Herrmann T, Jungbluth AA, et al. Cancer testis antigen expression in gastrointestinal stromal tumors: new markers for early recurrence. Int $\mathrm{J}$ Cancer 2008;123:1551-1555.

8 Perez D, Hauswirth F, Jäger D, et al. Protein expression of cancer testis antigens predicts tumor recurrence and treatment response to imatinib in gastrointestinal stromal tumors. Int J Cancer 2011;128:2947-2952. 